\title{
Perfil das Usuárias de Anticoncepcionais de Emergência: Uma Revisão Sistemática
}

\author{
Márcia Simone Almeida Galindo do Carmo ${ }^{1}$; Stênio Fernando Pimentel Duarte ${ }^{2}$
}

\begin{abstract}
Resumo: O uso de métodos contraceptivos tem aumentado, junto com a Contracepção de Emergência (CE). A anticoncepção de emergência é ofertada para mulheres em idade reprodutiva em situações de caráter excepcional. O uso da pílula do dia seguinte requer algumas precauções, com o intuito de garantir sua eficácia, dentre essas, destaca-se o intervalo de tempo da relação sexual e a sua administração. $O$ uso rotineiro compromete sua eficácia. Com isso, o estudo tem o intuito de avaliar o conhecimento das usuárias acerca do tema e agregar conhecimento sobre o mesmo. Metodologia: $\mathrm{O}$ estudo trata-se de uma revisão sistemática que reporta o uso de $\mathrm{CE}$, o mecanismo de ação, as vantagens e desvantagens de seu uso. A pesquisa científica será feita nos sites de busca Scientific Eletronic Library Online (SciELO), Literatura Latino-Americana e do Caribe em Ciências da Saúde (Lilacs) e Google Acadêmico, utilizando os seguintes descritores: contracepção de emergência, pílula do dia seguinte, reprodução, mecanismo de ação de pílula do dia seguinte, risco do uso da contracepção de emergência; que sejam atuais e na língua portuguesa, preferencialmente. Resultados e discussão: A idade média das mulheres participantes encontrados em estudos sobre uso de anticonceptivo de emergência (AE) foi de 21 anos e a classe econômica de baixa a média. A maioria dos estudos recomendam a administração desta medida contraceptiva de urgência em até 120 horas após a relação sexual. A falta de conhecimento das usuárias sobre atuação da pílula no organismo, bem como, dúvidas sobre os efeitos abortivos destes medicamentos, foram aspectos frequentemente abordados nos trabalhos encontrados. Considerações finais: O estudo sobre o uso de métodos contraceptivos de emergência é de extrema importância, haja vista a quantidade de mulheres que utilizam este método como contraceptivo. Desta forma, destaca-se a importância do acesso a informação e orientação de um profissional (farmacêutico), para que o desconhecimento dos usuários sobre os contraceptivos de emergência seja evitado.
\end{abstract}

Palavras-chave: Contracepção de emergência, Pílula do dia seguinte, Reprodução humana.

\section{Emergency Contraceptive Users Profile: A Systematic Review}

\begin{abstract}
The use of contraceptive methods has increased, along with Emergency Contraception (EC). Emergency contraception is offered to women of reproductive age in exceptional situations. The use of the morning-after pill requires some precautions, in order to guarantee its efficacy, among them, the time interval of the sexual relation and its administration stands out. Routine use compromises its effectiveness. With this, the study aims to evaluate the users' knowledge about the subject and to aggregate knowledge about it. Methodology: The study is a systematic review that reports the use of EC, the mechanism of action, the advantages and disadvantages of its use. Scientific research will be done on the Scientific Electronic Library Online (SciELO), Latin American and Caribbean Health Sciences (Lilacs) and Google Scholarly search sites, using the following descriptors: emergency contraception, morning-after pill, reproduction, Mechanism of action of morning-after pill, risk of use of emergency contraception; That are current and in the Portuguese language, preferably.Results and discussion: The mean age of the female participants found in studies on emergency contraceptive use (EC) was 21 years and the low- to middle-income class. Most studies recommend the administration of this emergency contraceptive measure within 120 hours after intercourse. The users' lack of knowledge about the action of the pill in the body, as well as doubts about the abortive effects of these drugs, were aspects frequently addressed in the works found. Final considerations: The study on the use of emergency contraceptive methods is extremely important, considering the number of women using this contraceptive method. In this way, the importance of access to information and guidance of a professional (pharmacist) is highlighted, so that the users' lack of knowledge about emergency contraceptives is avoided.
\end{abstract}

Keywords:Emergencycontraception, Next day'spill, Humanreproduction.

${ }^{1}$ Graduanda do Curso de Farmácia da Faculdade Independente do Nordeste (FAINOR). E-mail: simone_galindo1@hotmail.com;

${ }^{2}$ Graduação em Ciências Biológica, Mestrado e Doutorado em Fisiopatologia Clínica e Experimental pela Universidade do Estado do Rio de Janeiro - UERJ, atualmente é docente da Faculdade Independente do Nordeste e Faculdade Tecnologia e Ciências, Vitória da Conquista/BA. E-mail: steniofernando@gmail.com 


\section{Introdução}

O Ministério da Saúde (MS) define como Planejamento Familiar o direito sexual e reprodutivo que assegura a livre e responsável escolha de ter ou não filhos, recorrendo de ações que devem ser oferecidas pelo governo. Este direito é por sua vez assegurado na Constituição Federal e na Lei no 9.263, de 12 de Janeiro de 1996, que regulamenta o planejamento familiar (BRASIL, 1996; BRASIL, 2006).

O uso de métodos contraceptivos tem aumentado, junto com a Contracepção de Emergência (CE), que começou a ser disponibilizada no Brasil a desde 1999. Em 2000, o MS iniciou a oferta de contracepção de emergência para o atendimento às mulheres vítimas de violência sexual e a partir de 2002, passou também a fazer parte do Programa de Planejamento Familiar (BATAGLIÃO; MAMEDE, 2011). Desde então, o acesso a este método foi facilitado nas farmácias e drogarias, sendo poucas as mulheres que o utilizam perante prescrição médica (ALMEIDA et al., 2015).

No Brasil, a CE é regulamentada pelo MS, sendo também aprovada pela Agência de Vigilância Sanitária (ANVISA). Trata-se de um método contraceptivo que consiste na ingestão de uma substância hormonal, o levonorgestrel isolado, disponível em duas apresentações, $0,75 \mathrm{mg}$ (duas doses) e 1,5 mg (dose única), administrados após a relação sexual, no prazo máximo de até 120 horas, devendo ser utilizado sob prescrição médica, a fim de prevenir uma gravidez indesejada, sendo esta a forma mais efetiva de contracepção emergencial (DREZETT et al., 2011; ALANO et al., 2012; CHOFAKAIAN et al., 2014; WHO, 2016).

Segundo a Organização Mundial de Saúde (OMS), o mecanismo de ação deste método oscila de acordo com o ciclo menstrual em que a mulher se encontra, podendo atuar na primeira fase do ciclo, no retardo ou no impedimento da ovulação, tendo ação também na segunda fase, através da modificação na viscosidade do muco cervical, alterando a motilidade dos espermatozoides e consequentemente, impedindo a fertilização (WHO, 2016).

A anticoncepção de emergência (AE) ou "pílula do dia seguinte", como é conhecida popularmente, é ofertada para mulheres em idade reprodutiva, respeitando às diferentes faixas etárias, em situações de caráter emergencial, como em relações sexuais desprotegidas, não uso ou falha de preservativos e em casos de violência sexual (BRASIL, 2010(a); CHOFAKAIAN et al., 2014).

O uso da pílula do dia seguinte requer algumas precauções, com o intuito de garantir sua eficácia, dentre essas, destaca-se o intervalo de tempo entre a relação sexual e a sua administração, intervalo este que pode ocorrer em até 120 horas, porém, preferencialmente até às 72 horas após o coito desprotegido, visto que quanto mais rápido for a sua administração, maior será sua eficácia. $\mathrm{O}$ uso rotineiro compromete sua eficácia (BORGES et al., 2010; LUPIÃO; OKAZAKI, 2011; WHO, 2016).

O uso somente em emergências é justificado pelo fato de se tratar de um método que não oferece nenhuma proteção contra as infeções sexualmente transmissíveis, bem como a existência de outros métodos 
contraceptivos adequados para o uso contínuo, que possua efeitos adversos diminuídos (ALANO et al., 2012).

A CE quando utilizada com cautela e sob prescrição médica não oferece riscos importantes à saúde da mulher, ajudando a diminuir o número de abortos e gravidez indesejada. Ao passo que, quando utilizada exacerbadamente, além de levar a diminuição da eficácia, pode desencadear problemas hormonais ou mesmo de infertilidade (ALMEIDA et al., 2015).

A busca para ampliação do acesso a anticonceptivo de emergência (AE) está ligada ao interesse em diminuir as taxas de gravidez na adolescência, gravidez imprevista e aborto inseguro, por isso alguns países desenvolvidos têm formulado políticas para melhorar o acesso a esses métodos (PAIVA; BRANDÃO, 2012).Uma questão frequentemente levada a debate é de como ampliar a oferta à informação para que estas mulheres tenham acesso ao AE. Pois, sabe-se que atualmente o medicamento é vendido cotidianamente nas farmácias sem controle algum, sem prescrição e acompanhamento médico,com o mínimo de conhecimento e informações que não esclarecem osriscos à saúde das usuárias(PAIVA; BRANDÃO, 2012).

Diante deste cenário, o estudo em questão teve por objetivo realizar uma revisão sistemática através da literatura científica sobre o tema contracepção de emergência, com intuito de avaliar o conhecimento das usuárias acerca do tema e agregar conhecimento sobre o mesmo, o que é absolutamente desejável, visto que tal método se apresenta como uma estratégia contraceptiva que deve ser ofertada, sendo amplamente utilizado, e de extrema importância para a saúde da mulher.

\section{Metodologia}

A pesquisa consiste em uma revisão sistemática que reporta o uso de contraceptivos de emergência, o mecanismo de ação desta substância e também as vantagens e desvantagens de seu uso.

Para a estruturação desse estudo, foram realizadas buscas de conhecimento disponível em artigos científicos, livros, diretrizes, bem como manuais, tendo como fonte de pesquisa a literatura científica nos sites de busca Scientific Eletronic Library Online (SciELO), Literatura Latino-Americana e do Caribe em Ciências da Saúde (Lilacs) e Google Acadêmico.

Foram utilizados, para consulta às bases de dados, os seguintes descritores: contracepção de emergência, pílula do dia seguinte, reprodução, mecanismo de ação de pílula do dia seguinte, risco do uso da contracepção de emergência. Para compor a revisão do tema foram adotados critérios de inclusão, os quais deveriam possuir temas relevantes relacionados com as palavras-chaves, bem como atuais, dos últimos 7 anos e também aqueles em que se encontravam de preferência na língua portuguesa.

Ao final do levantamento bibliográfico, somando-se todas as bases de dados, foram efetivamente utilizados 20 instrumentos, dentre eles, artigos, diretrizes, livros, que preenchiam os critérios inicialmente propostos. Que abrangeu o período compreendido entre a 2010 a 2017. Os resultados foram organizados em 
Id on Line Revista Multidisciplinar e de Psicoloqia

Id on Line Multidisciplinary and Psycology Journal

tópicos e estão dispostos nos resultados e discussão desse estudo.O período em que ocorreu tal levantamento bibliográfico para a realização da revisão de literatura, ficou compreendido no mês de abril e maio do ano corrente.

\section{Resultados e Discussão}

Alguns artigos utilizados para compor este trabalho foram dispostos na tabela 1, indicados por autores, ano de desenvolvimento e síntese.Após serem lidos e comparados, algumas variáveis foram organizadas e selecionadas, com intuito de contrapor as opiniões relevantes dos autores sobre o uso de anticoncepcionais de emergência.

Tabela 1 Síntese dos artigos sobre tempo ideal para administração, fatores associados, perfil das usuárias e conhecimentos sobre a ação dos CE, que foram utilizados para estruturação do trabalho

\begin{tabular}{|c|c|c|}
\hline Autores & Ano & Síntese \\
\hline DE ALMEIDA et al., & 2015 & $\begin{array}{l}\text { O contraceptivo de emergência deve ser utilizado até } 72 \text { horas após relação } \\
\text { sexual. }\end{array}$ \\
\hline DREZETT et al., & 2011 & $\begin{array}{l}\text { A administração do contraceptivo de emergência pode ser feita em até } 5 \text { dias } \\
\text { após a relação sexual. }\end{array}$ \\
\hline LUPIÃO; OKAZAKI, & 2011 & $\begin{array}{l}\text { O contraceptivo de emergência pode ser administrado até cinco dias após a } \\
\text { relação sexual desprotegida. }\end{array}$ \\
\hline BRANDÃO et al., & 2016 & $\begin{array}{l}\text { A contracepção de emergência, conhecida popularmente como "pílula do dia } \\
\text { seguinte", é um contraceptivo utilizado em situações de emergência, que deve } \\
\text { ser administrado em até } 120 \text { horas para se evitar uma gravidez indesejada. }\end{array}$ \\
\hline HEVIA, & 2012 & $\begin{array}{l}\text { Revisões de literatura na América Latina sobre CE, recomenda, para que se } \\
\text { tenho o efeito desejado, o prazo de até } 120 \text { horas para administração da pílula, } \\
\text { mas preferencialmente até } 72 \text { horas. }\end{array}$ \\
\hline BORES et al., & 2010 & $\begin{array}{l}\text { Faixa etária, idade na iniciação sexual, não ter usado ou falha do preservativo } \\
\text { nas relações sexuais e conhecimento de alguém que usou CE foram } \\
\text { associados ao uso destas pílulas. }\end{array}$ \\
\hline $\begin{array}{l}\text { TILAHUN; ASSEFA; } \\
\text { BELACHEW, }\end{array}$ & 2010 & $\begin{array}{l}\text { O pouco conhecimento sobre } \mathrm{AE} \text {, os extremos etários (menores de } 20 \text { e } \\
\text { maiores de } 40 \text { anos), a baixa escolaridade, já ter tido filho e ter vivenciado } \\
\text { abortamento prévio são fatores associados ao não uso desses } \mathrm{AE} \text {. }\end{array}$ \\
\hline CAVALCANTE et al, & 2016 & $\begin{array}{l}\text { A maioria das usuárias de pílulas do dia seguinte concentrou-se na faixa etária } \\
\text { adolescentes e jovens, com baixa ou médio poder econômico e que iriam usar } \\
\text { o CE sem orientação e prescrição de um profissional da saúde, sendo as } \\
\text { informações sobre a pílula oriundas de familiares, amigos e mídia. }\end{array}$ \\
\hline $\begin{array}{l}\text { DE MORAIS } \\
\text { PEREIRA, }\end{array}$ & 2010 & $\begin{array}{l}\text { O mecanismo de ação destes métodos ainda é motivo de controvérsias. } \\
\text { Embora existam estudos sobre o tema, ainda há pouco conhecimento sobre o } \\
\text { mesmo, principalmente por partes das pacientes e alguns profissionais da } \\
\text { saúde. Uma das principais dúvidas, é sobre o efeito abortivo. Com isto, é de } \\
\text { extrema importância o estudo do uso correto da pílula por estes profissionais } \\
\text { para esclarecimento de eventuais dúvidas de seus pacientes. }\end{array}$ \\
\hline PAIVA; BRANDÃO, & 2012 & $\begin{array}{l}\text { A falta de conhecimento das usuárias sobre atuação da pílula no organismo, } \\
\text { bem como, dúvidas sobre os efeitos abortivos destes medicamentos, foram } \\
\text { aspectos frequentemente abordados nos trabalhos encontrados. }\end{array}$ \\
\hline
\end{tabular}

Fonte: Pesquisa própria, 2017. 
Tendo em vista que valores de prevalência podem sofrer variação de acordo a faixa etária pesquisada e delineamento da pesquisa, em estudos analisados, a idade média das mulheres usuárias de AE foi de 21 anos, com variação entre 18 a 46 anos e a frequência do uso foi em média 2,4 vezes. A classe econômica com mais utilização de AE foi a classe B (BATAGLIÃO, 2011; ALANO et al., 2012). Um estudo evidenciou uma proporção de 48,6\% de mulheres que utilizavam esses métodos. Em São Paulo, outro estudo realizado com universitárias encontrou que 50,4\% das mulheres participantes, relataram fazer uso de AE (BORGES et al., 2010; ALANO et al., 2012).

Estudos atuais, relatam quea maioria das usuárias de $\mathrm{AE}$ encontram-se na faixa etária de adolescentes e jovens. A maioria dessas usuárias têm baixo ou médio poder econômico e utilizavam a pílula do dia seguinte sem qualquer orientação ou prescrição de um profissional da saúde. Tais informações sobre o uso destes medicamentos eram oriundos de amigos, familiares e mídia, o que causa extrema preocupação, visto o fácil acesso nas farmácias comerciais e eventuais riscos com o uso inadequado (CAVALCANTE et al., 2016).

Constatou-se em um estudo com mulheres universitárias, que quanto menor a idade, maior a prevalência de utilização do método ( $\mathrm{p}=0,02961)$. Não foi encontrada associação significativa entre usar CE ou a frequência deste uso com as demais variáveis obtidas no estudo (ALANO et al., 2012). Corroborando com este estudo, BORGES et al., 2010, evidenciou ainda que faixa etária, idade na iniciação sexual, não ter usado ou falha do preservativo nas relações sexuais e conhecimento de alguém que usou CE foram associados ao uso destas pílulas.Em contrapartida, outros autores têm também enfatizado que o baixo nível de conhecimento sobre $\mathrm{CE}$, os extremos etários,menor escolaridade, já ter filho eexperiência prévia de aborto são ainda associados ao não uso da AE (TILAHUN, 2010).

A maior parte das mulheres demonstraram ter conhecimento sobre o uso de AE (BATAGLIÃO, 2011) e análises de regressão mostraram que o tipo de escola, idade, sexo, início da vida sexual, uso prévio da $\mathrm{AE}$ e conhecer alguém que já utilizou associaram-se com o conhecimento sobre esses medicamentos na adolescência (CHOFAKIAN, 2014). No entanto, apesar de uma grande parcela dos estudos se realizarem com mulheres universitárias, $15 \%$ das participantes desconheciam o fato da pílula do dia seguinte não prevenir doenças sexualmente transmissíveis (DST), dado preocupante haja vista que se tratamde mulheres envolvidasno meio acadêmico, e com grande acesso às informações (ALANO et al., 2012).

Um estudo sobre o uso e conhecimento de CE em adolescentes, evidenciou um baixo uso de CE $(28,8 \%)$ sendo a primeira utilização aos 14 anos e com uso em média de 3 vezes. Também foi questionado às adolescentes sobre dúvidas acerca desses medicamentos e 32,5\%tinham dúvida sobre a eficácia, utilização correta e diminuição de sua eficácia com o uso contínuo, bem como, os efeitos e riscos para à saúde. Atualmente no Brasil, são comercializados dois tipos de contraceptivos de emergências, os de dose única e os que dividem a carga hormonal em dois comprimidos, sendo que o segundo deve ser tomado com intervalo de 12 horas após o primeiro (SOUZA; BRANDÃO 2009), no entanto, uma parcela expressiva de adolescentes desconhecem esta informação que, se descumprida, compromete a eficácia do método (RODRIGUES; 
JARDIM, 2012). Os CE devem ser usados em situações específicas e isto parece ser conhecidopor quasetodosos adolescentes usuários. Contudo, o uso abusivo e o impacto desses métodos na rotina de contraceptivos contínuos é uma grande preocupação dos profissionais de saúde que atendem adolescentes visto a de diminuição ou abandono do uso de preservativo e exposição às DST/Aids (RODRIGUES; JARDIM, 2012).

Em outro estudo com estudantes, não houve participante que demostrasse conhecimento sobre o tempo máximo que a AE pode ser administrada (120 horas). Menos da metade da amostra respondeu que o tempo máximo é menor que o indicado (72 horas) e quase metade respondeu que o uso máximo é de 24 a 48 horas após a relação sexual desprotegida, possivelmente pelo nome popular dos CE persuadir os adolescentes, fazendo com que eles achem que o uso é indicado apenas no dia seguinte ao coito desprotegido (SILVA et al., 2010). Como citado na tabela 1, vários autores preconizam que o uso do contraceptivo de emergência seja realizado em até 120 horas após relação sexual (DREZETT et al., 2011; LUPIÃO; OKAZAKI, 2011; DE ALMEIDA et al., 2015; BRANDÃO et al., 2016), mas preferencialmente até 72 horas (HEVIA, 2012), visto que maior é sua efetividade quando mais cedo é administrado em uma situação de emergência, como, por exemplo, na falha do preservativo ou em uma violação sexual (DE MORAIS PEREIRA, 2010).

É de extrema importância que esse método não seja a escolha para contraceptivo contínuo na intenção de controle da natalidade, visto que este é menos efetivo que outras formas indicadas para contracepção de uso regular, além de não proteger contra doenças sexualmente transmissíveis (DE MORAIS PEREIRA, 2010).

O mecanismo de ação desses contraceptivos no organismo feminino ainda é motivo de controvérsias(DE MORAIS PEREIRA, 2010). O hormônio utilizado para composição das pílulas contraceptivas de emergência age em vários sítios, tendo ação sobre o eixo hipotalâmico-hipofisárioovariano, interfere no preparo do endométrio através de moléculas dependentes de progesterona propiciando uma má implantação do óvulo, interfere na mobilidade da tuba uterina, altera a última fase de maturação dos espermatozóides inseridos no organismo da mulher, interfere também no $\mathrm{pH}$ do fluido uterino e viscosidade do muco cervical (ZUCCHI et al., 2004). O conhecimento sobre estes mecanismos e formas de ação por parte das pacientes, e também dos profissionais da saúde, ainda é relativamente escasso. Dúvidas são frequentes, principalmente sobre o risco de efeito abortivo. Portanto, o conhecimento aprofundado sobre estes contraceptivos é de fundamental importância para os profissionais de saúde, visto que é de grande valia o esclarecimento quanto aos efeitos dos AE para as suas pacientes (DE MORAIS PEREIRA, 2010).

A falta de conhecimento das usuárias sobre atuação da pílula no organismo, bem como, dúvidas sobre os efeitos abortivos destes medicamentos, foram aspectos frequentemente abordados nos trabalhos encontrados. Propostas para uma educação sexual voltada aos adolescentes e jovens devem ser debatidas (PAIVA; BRANDÃO, 2012). Com tudo, espera-se que o conhecimento adquirido sobre anticoncepcionais 
de emergêncialeve a uma utilização mais segurados mesmos e que o uso de métodos de barreiraseja estimulado (DE MORAIS PEREIRA, 2010).

\section{Considerações Finais}

Nossa revisão concluiu que há poucos artigos sobre o perfil do uso destes medicamentos no Brasil. Com isso, estudos sobre o uso de métodos contraceptivos de emergência é de extrema importância, haja vista a quantidade de mulheres que utilizam este método como contraceptivo. Grande parte dos estudos recomendam o uso desta medida contraceptiva de urgência em até 120 horas após a relação sexual, no entanto, alguns estudos afirmam que o quanto antes a pílula for administrada, mais eficaz será a prevenção de uma gravidez indesejada. Os fatores mais associados ao uso foram a idade, idade ao início da vida sexual, não ter usado ou ocorrência de falhados preservativos nas relações sexuais e conhecimento de alguém que usou CE.

Desta forma, destaca-se a importância do acesso a informação e orientação de um profissional (farmacêutico), para que o desconhecimento dos usuários sobre os contraceptivos de emergência seja evitado, diminuindo assim o uso inadequado, riscos, desconhecimento sobre modo de atuação no organismo feminino e confusão com outros medicamentos abortivos.

\section{Referências}

ALANO, G. M. et al. Conhecimento, consumo e acesso à contracepção de emergência entre mulheres universitárias no sul do Estado de Santa Catarina. CienSaudeColet, v. 17, n. 9, p. 2397-404, 2012.

BATAGLIÃO, E. M. L. et al. Conhecimento e utilização da contracepção de emergência por acadêmicos de enfermagem. Esc Anna Nery, v. 15, n. 2, p. 284-90, 2011.

BRASIL. Lei n ${ }^{\circ} 9.263$, de 12 de Janeiro de 1996. Regula o $\$ 7^{\circ}$ do art. 226 da Constituição Federal, que trata do regulamento familiar estabelece penalidades e dá outras providências. Diário Oficial da República Federativa do Brasil. Brasília, 1996.

. MINISTÉRIO DA SAÚDE, SECRETARIA DE ATENÇÃO À SAÚDE. Departamento de Aç̃̃es Programáticas Estratégicas. Direitos sexuais, Direitos Reprodutivos e Métodos Anticoncepcionais. BRASÍLIA, 2006.

. MINISTÉRIO DA SAÚDE, COMISSÃO DE CIDADANIA E REPRODUÇÃO. Contracepção de Emergência no Brasil e na América Latina: Dinâmicas Políticas e Direitos Sexuais e Reprodutivos. São Paulo, Oficina Editorial, 2010. 
BORGES, A. L. V. et al. Práticas contraceptivas entre jovens universitários: o uso da anticoncepção de emergência. Cadernos de Saúde Pública, v. 26, n. 4, p. 816-826, 2010.

BRANDÃO, E. R. et al. " Hormone bomb": risks of emergency contraception from the perspective of pharmacy attendants in Rio de Janeiro, Brazil. Cadernos de Saúde Pública, v. 32, n. 9, 2016.

CAVAlCANTE, M. S. et al. Perfil de utilização de anticoncepcional de emergência em serviços de atendimento farmacêutico de uma rede de farmácias comunitárias. Revista Eletrônica de Farmácia, v. 13, n. 3, p. 131-139, 2016.

CHOFAKIAN, C. B. N. et al. Conhecimento sobre anticoncepção de emergência entre adolescentes do Ensino Médio de escolas públicas e privadas. Cad Saúde Pública, p. 1525-1536, 2014.

DE ALMEIDA, F. B. et al. Avaliação do Uso de Anticoncepcionais de Emergência entre Estudantes Universitários. Revista Brasileira de Educação e Saúde, v. 5, n. 3, p. 49-55, 2015.

DE MORAIS PEREIRA, S. Rompendo preconceitos sobre a utilização da anticoncepção de emergência para as adolescentes. Adolescencia e Saude, v. 7, n. 1, p. 31-36, 2010.

DREZETT, J. et al. Mecanismo de ação da anticoncepção de emergência. ReprodClim, v. 26, p. 44-51, 2011.

HEVIA, M. The legal status of emergency contraception in Latin America. InternationalJournalofGynecology\&Obstetrics, v. 116, n. 1, p. 87-90, 2012.

LUPIÃO, A. C.; OKAZAKI, E. Métodos anticoncepcionais: revisão. Revisão. Rev, 2011.

PAIVA, S. P.; BRANDÃO, E. R. Contracepção de emergência no contexto das farmácias: revisão crítica de literatura. Physis: Revista de Saúde Coletiva, v. 22, n. 1, 2012.

RODRIGUES, Milena Freitas; JARDIM, Dulcilene Pereira. Conhecimento e uso da contracepção de emergência na adolescência: contribuições para a enfermagem. Cogitare Enfermagem, v. 17, n. 4, 2012.

SILVA, F. C. et al. Diferenças regionais de conhecimento, opinião e uso de contraceptivo de emergência entre universitários brasileiros de cursos da área de saúde. Cadernos de SaúdePública, 2010.

TILAHUN, D.; ASSEFA, T.; BELACHEW, T. Predictors of emergency contraceptive use among regular female students at Adama University, Central Ethiopia. Pan African Medical Journal, v. 7, n. 16, 2010.

WORLD HEALTH ORGANIZATION. Media Center. Emergency Contraception. Fact sheet n⿳0 244, 2016. Disponível em: <http://www.who.int/mediacentre/factsheets/fs244/en/> Acesso em: 15 mai 2017.

ZUCCHI, R. M. et al. Gravidez ectópica após uso de contracepção de emergência: relato de caso. RBGO, v. 26, n. 9, 2004.

\section{Como citar este artigo (Formato ABNT):}

DO CARMO, Márcia Simone A. G.; DUARTE, Stênio F P Perfil das Usuárias de Anticoncepcionais de Emergência: Uma Revisão Sistemática. Id on Line Revista Multidisciplinar e de Psicologia, Maio de 2017, vol.11, n.35, p. 317-324. ISSN: 1981-1179.

Recebido: 25.05.2017

Aceito: 26.05 .2017 